\title{
Taking inventory with shotgun EM
}

The combination of single-particle electron microscopy and mass spectrometry shows potential for surveys of both the structure and the identity of protein complexes in the cell.

$\mathrm{S}$ ometimes, two methods are better than one. Mass spectrometry (MS)-based proteomics excels at taking inventory of cellular proteins. Single-particle electron microscopy (EM) provides structural information about purified protein complexes. Putting these established methods together can provide even more power for exploring the biochemistry of a cell, as David Taylor, Edward Marcotte and their colleagues demonstrated recently in developing an approach they call 'shotgun EM'.

Though still at a proof-of-concept stage, their work illustrates a way to determine both identities and structures of unknown, native protein complexes in a cell lysate, at a potentially high-throughput level. In a typical protein-structure study, you need to "purify the heck out of something," Taylor notes. "It's time consuming and not always guaranteed to work." With shotgun EM, he sees strong advantages in using minimal sample preparation.

Taylor, who started his lab at the University of Texas at Austin just two years ago, began pursuing the project with the support of new investigator funding for a high-risk project. He wasn't sure it would work, but he had a tenacious graduate student willing to go for it.

The method involves the use of sizeexclusion chromatography to separate a human cell lysate into different molecularweight fractions. The team focused on the high-molecular-weight fraction ranging from 1.5 to 2 megadaltons, which is likely to contain protein complexes. Using MS, they identified 1,401 unique proteins. They mapped these proteins to human proteininteraction network resources to narrow down the possible identities of complexes that were likely to be present in this fraction.

In parallel, they carried out negative stain EM to obtain structure information for complexes in the high-molecular-weight fraction. They computationally filtered out the 'junk' particles and sorted the remaining particles into different homogeneous $2 \mathrm{D}$ classes based on complex shape, then used the sorted data to generate reconstructions

of 30 distinct complexes. Among their topscoring structures, they were able to readily identify the barrel-shaped 20 S proteasome complex, whose presence was supported by the MS data; they also observed a singlecapped proteasome. One of their top-scoring structures, however, was not recognizable from its shape, and they were unable to conclusively determine its identity even after cross-correlation with the MS data.

Taylor hopes that the higher resolution made possible by cryo-EM will make it much easier to identify protein complexes with this kind of approach. At the relatively low resolution offered by negative stain EM, "it's very difficult to identify what they are," he says. The team began with negative stain EM because of its technical simplicity and good signal-to-noise ratio. For all of its advantages, cryo-EM comes with many technical complications, including the difficulties of freezing protein complexes on sample grids. Other experimental refinements, such as the use of simpler (non-human) cellular systems, finer separations to generate fractions of lower complexity, and a gentler fractionation approach to preserve fragile assemblies, also will be likely to improve their ability to identify more complexes.

Taylor and his team also plan to further refine their computational analysis by developing improved algorithms for sorting the particles in the raw EM images into distinct shape classes, and by using protein-structure interface prediction and computational docking methods to help identify complexes in their EM data. It will be interesting to see whether such improvements in these areas facilitate broad adoption of 'shotgun EM'.

\section{Allison Doerr}

Published online: 31 August 2018

https://doi.org/10.1038/s41592-018-0132-x

Research papers

Verbeke, E. J. et al. Classification of single particles from human cell extract reveals distinct structures. Cell Rep. 24, 259-268 (2018).

\section{SARSTEDT}

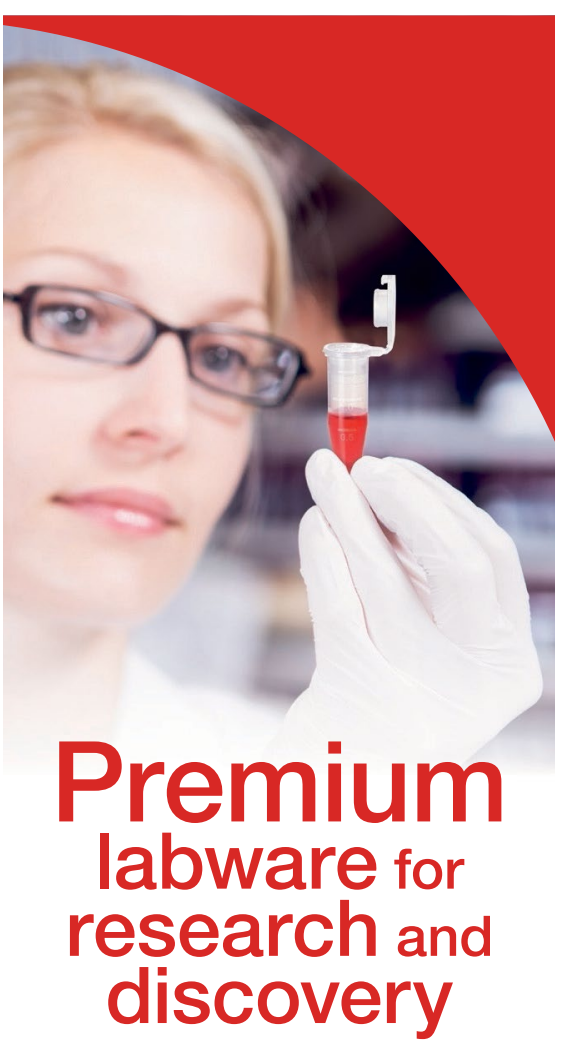

Low-Binding Micro Tubes

- Minimal DNA / protein loss

- SafeSeal locking cap

- Certified PCR-Performance Tested

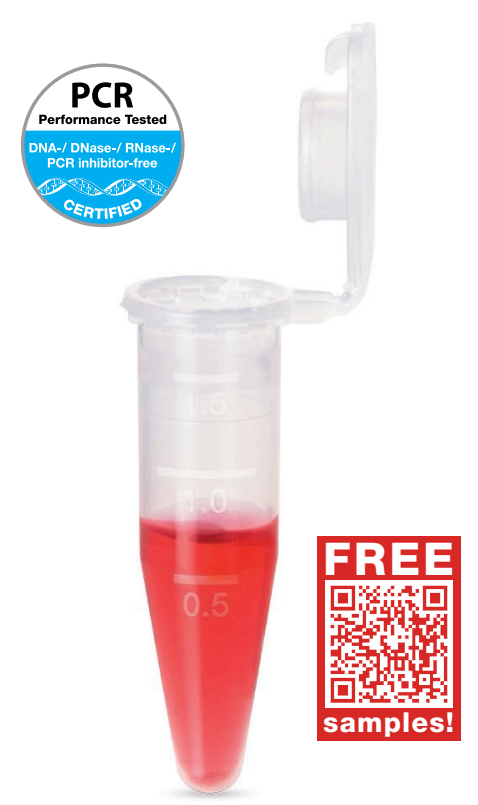

www.sarstedt.com 